\title{
Degradation of cyflumetofen and formation of its main metabolites in soils and water/sediment systems
}

\author{
Pingping Wang ${ }^{1} \cdot$ Minmin $\mathrm{Li}^{2}$ • Xingang Liu ${ }^{1}$ Jun $\mathrm{Xu}^{1}$ • Fengshou Dong ${ }^{1}$. \\ Xiaohu $\mathrm{Wu}^{1} \cdot$ Yongquan Zheng ${ }^{1}$
}

Received: 18 April 2016 / Accepted: 26 August 2016 / Published online: 3 September 2016

(C) Springer-Verlag Berlin Heidelberg 2016

\begin{abstract}
Cyflumetofen is a novel benzoyl acetonitrile acaricide without cross-resistance to existing acaricides. In the present study, for the first time, the environmental behaviors of cyflumetofen and the formation of its main metabolites, 2-(trifluoromethyl) benzoic acid (B-1) and 2-(trifluoromethyl) benzamide (B-3), in the four types of soil (black soil, sierozem, krasnozem, and fluvo-aquic soil) and three types of water/sediment systems (Northeast Lake, Hunan paddy field, and Beijng Shangzhuang reservoir) under aerobic and anaerobic conditions were investigated. The degradation dynamics of cyflumetofen followed first-order kinetics. Under aerobic environment, the half-lives of cyflumetofen in black soil, sierozem, krasnozem and fluvo-aquic soil were 11.2, $10.3,12.4$, and 11.4 days. Under water anaerobic conditions, the half-lives were 13.1, 10.8, 13.9, and 12.8 days. The effects of different conditions and soil types on the half-lives of cyflumetofen were studied using a one-way ANOVA test with post hoc comparison (Tukey's test). It was shown that the differences in black soil, krasnozem, and fluvo-aquic soil were extremely significant difference $(p<0.05)$ under aerobic and water anaerobic conditions. And there is a strong correlation between half-life and $\mathrm{pH}$. Under aerobic environment, the
\end{abstract}

Responsible editor: Ester heath

Pingping Wang and Minmin Li contributed equally to this work.

Xingang Liu

liuxingang@caas.cn

1 State Key Laboratory for Biology of Plant Diseases and Insect Pests, Institute of Plant Protection, Chinese Academy of Agricultural Sciences, Beijing 100193, People's Republic of China

2 Institute of food science and technology CAAS, Chinese Academy of Agricultural Sciences, Beijing 100193, People's Republic of China half-lives of cyflumetofen in Northeast Lake, Hunan paddy field, and Beijng Shangzhuang reservoir were 15.4, 16.9, and 15.1 days. Under anaerobic conditions, they were 16.5, 17.3, and 16.1 days. Analyzing the differences of the halflives under aerobic and anaerobic conditions, the difference only in Shangzhuang reservoir was extremely significant difference $(p<0.05)$. In soils, cyflumetofen degraded metabolites B-1 and B-3, from the first day $0.24 \%$ B-1 was generated, while, only very low levels of B-3 generated at the same time. As time increased, B-3 gradually increased, cyflumetofen reduced gradually. Until 100 days, there were about $3.5 \%$ B-1 and B-3 in the soils. In the water/sediment systems, from the first day, it degraded into B-1 in the sediment, and in the water mainly degraded into B-3.

Keywords Cyflumetofen $\cdot$ Metabolites $\cdot$ Soils $\cdot$ Water/ sediment $\cdot$ Degradation

\section{Introduction}

Pesticides are the most cost-effective means of pest and weed control, and widespread in the environment (AriasEstévez et al. 2008, Fenner et al. 2013). In some, their use has increased during the past few years (Dalvie et al. 2009) despite their well-known hazards for environment and human health (Bermúdez-Couso et al. 2011, Everett \& Matheson 2010, McKinlay et al. 2008, Seiber \& Kleinschmidt 2011). It has been estimated that less than $0.1 \%$ of the pesticide actually reaches the target, the rest enters the environment (Pimentel 1995, Pimentel \&Levitan 1986). Pesticides and their bioactive metabolites accumulate and induce adverse effects in soils and sediments (Babić et al. 2007, Díaz-Cruz et al. 2003). With increasing knowledge of their environmental 
occurrence, more interest was focused on their environmental fate (Löffler et al. 2005). Nevertheless, limited data were available covering the fate of the pesticides in the soil and water/sediment. Therefore, evaluation of the pesticides degradation and its main metabolites formation in soils and water/sediment systems is needed to better predict the chemical lifetime in the environment and to accurately assess potential biological impacts.

Cyflumetofen is a relatively novel acaricide developed by Otsuka AgriTechno Co., Ltd. in 2007, and now used in 15 countries around the world (Hayashi et al. 2013). Cyflumetofen is a mitochondrial electron transport inhibitor pesticide and has been recognized as a suitable acaricide for integrated pest management (IPM) systems without crossresistance to existing acaricides (Marcic 2012). Researches have demonstrated that cyflumetofen has highly selective efficacy (Yoshida et al. 2012). It is against a variety of mites, and the efficacy was not affected by the developmental stages of mites, temperature, or any other field conditions (Takahashi et al. 2012).

Data of the cyflumetofen residues in the environment are mostly limited to the parent compound, but metabolites of cyflumetofen are often released into the environment in higher quantities than their parent compound ( $\mathrm{Li}$ et al. 2013, Li et al. 2012). According to EFSA, the metabolites B-1 (2-(trifluoromethyl) benzoic acid), B-2 $(\alpha, \alpha, \alpha$-trifluoro-o-toluic anhydride), B-3 (2-(trifluoromethyl) benzamide), and AB-1 (2-(4-tertbutylphenyl)-3-oxo-3-( $\alpha, \alpha, \alpha$-trifluoro-otolyl) propriononitrile) were formed in the soil and water/sediment. AB-1 was essentially immobile in soil, and it was considered that AB-1 is not a major soil metabolite, B-1 and B-3 exhibited very high mobility. From the toxicological point of view, B-3 appeared to be more toxic than its parent compound (Authority 2012). The information on the degradation of cyflumetofen and the formation of B-1 and B-3 in Chinese soils and water/sediment systems under aerobic and anaerobic conditions are great significance. In this study, B-1 and B-3 as the main metabolites (Table 1), and a study was investigated to understand what controls the environmental degradation of cyflumetofen using ultrahigh performance liquid chromatography-tandem mass spectrometry (UPLC-MS/ MS) for analysis. The half-life period $\left(T_{1 / 2}\right)$ of cyflumetofen in four soils and three water/sediment systems was investigated. The research will be relevant for assessing the cyflumetofen degradation processes in the field and anticipating the pathways of these compounds' degradation under specific field conditions. And it is the first time to investigate the degradation of cyflumetofen and formation of its main metabolites in four soils and three water/sediment systems under aerobic and anaerobic conditions.

\section{Materials and method}

\section{Chemicals and reagents}

The analytical standard cyflumetofen (purity $99.8 \%$ ) and its metabolites, B-1 and B-3, were obtained from Otsuka AgriTechno Co., Ltd. (Osaka, Japan). Chromatography grade acetonitrile and methanol for HPLC analysis were obtained from Honeywell International Inc. (New Jersey, USA). Analytical grade acetonitrile for pesticide residue analysis was purchased from Beijing Chemical Reagent Company (Beijing, China). Sodium chloride $(\mathrm{NaCl})$ and anhydrous magnesium sulfate (anhydrous $\mathrm{MgSO}_{4}$ ) were analytical grade and purchased from Beijing Chemical Company (Beijing, China). MWCNTs (multi-walled carbon nanotubes) with different average external diameters $(<8 \mathrm{~nm})$ were purchased from Boyu Technologies Inc. (Beijing, China). Ultrapure water for HPLC was prepared using a Milli-Q water purification system (Bedford, MA, USA).

\section{Test soils and water/sediments}

The four different agricultural soils representing different physicochemical properties and climatic environments were obtained from the $0-15-\mathrm{cm}$ surface layer in fields from four distinct sites in China: Ha'erbin (Black soil) in the Heilongjiang Province, Langfang (Fluvo-aquic soil) in the Hebei Province, Changsha (Krasnozem) in the Hunan Province, and Xi'an (Sierozem) in the Shanxi Province. The soils were air dried at room temperature and passed through a $1-\mathrm{mm}$ sieve to remove pebbles and plant debris. The moisture content of the soils was adjusted to $60 \%$ of the water-holding capacity (WHC) using deionized water, and samples were kept in the dark at $25 \pm 2{ }^{\circ} \mathrm{C}$ for a 2-week activation period.

Sediment samples with associated water were collected in the anaerobic zone of surface water bodies from Shangzhuang reservoirs (Beijing), Hunan paddy fields (Changsha), and Ha'erbin (Heilongjiang), using a clean methanol rinsed stainless steel trowel. The sediments were sampled from the top layer of sediment and sieved with a 2-mm screen following the OECD 308 guideline (OECD 2002). Associated waters were taken from the same location. The anaerobic sediments were sampled to exclude oxygen and stored at approximately $4{ }^{\circ} \mathrm{C}$. The basic physicochemical characteristics of test soils and sediments were given and summarized in Table 2.

\section{Soils and water/sediments novel incubation under aerobic/anaerobic conditions}

The removable nitrogen circulation anaerobic culture device comprised a nitrogen generator, incubator, and nitrogen blowing instrument. Among them, the intake of the incubator tube and nitrogen gas phase connected with the air outlet pipe 
Table 1 Properties of cyflumetofen and metabolites

\begin{tabular}{|c|c|c|c|}
\hline Property & cyflumetofen & B-1 & B-3 \\
\hline $\begin{array}{l}\text { Chemical } \\
\text { structure }\end{array}$ & & & \\
\hline Molecular mass & 447.45 & 190.12 & 189.13 \\
\hline $\begin{array}{c}\text { Molecular } \\
\text { formula }\end{array}$ & $\mathrm{C}_{24} \mathrm{H}_{24} \mathrm{~F}_{3} \mathrm{NO}_{4}$ & $\mathrm{C}_{8} \mathrm{H}_{5} \mathrm{~F}_{3} \mathrm{O}_{2}$ & $\mathrm{C}_{8} \mathrm{H}_{6} \mathrm{~F}_{3} \mathrm{NO}_{4}$ \\
\hline $\begin{array}{c}\text { Solubility in } \\
\text { water at } 20^{\circ} \mathrm{C} \\
(\mathrm{mg} / \mathrm{L})\end{array}$ & 0.028 & - & 13000 \\
\hline $\log \mathrm{P}$ & 4.3 & - & 0.68 \\
\hline $\mathrm{K}_{\mathrm{d}}$ & 2090 & 1.8 & - \\
\hline $\begin{array}{l}\text { UPLC retention } \\
\text { times }(\mathrm{min})\end{array}$ & 3.37 & 1.66 & 1.20 \\
\hline
\end{tabular}

device, cultivation box out ventilation trachea and pipe of nitrogen blowing instrument. Nitrogen-generating device was communicated with each individual anaerobic culture vessels through the plastic pipe, to the nitrogen circulation, and through the three-way valve controlled the nitrogen flow rate at any time. When the training was completed, either anaerobic culture vessels can be taken out at any times without affecting the other environment culture vessel. The culture device represented the stricter anaerobic environment.

One set of soil samples was used for incubation under aerobic conditions. Blank determination of the soils revealed no cyflumetofen, B-1 and B-3. After pre-incubating for 14 days, a portion of the soil (20 g) was spiked with
$1000 \mathrm{mg} \mathrm{L}^{-1}$ cyflumetofen acetone stock solution and stirred for $5 \mathrm{~min}$. The spiked soil was allowed to air-dry $(10 \mathrm{~min})$ until the solvent was completely evaporated, and the final concentration in the soils was approximately $10 \mathrm{mg} \mathrm{kg}^{-1}$ (dry weight). The water content of the soil samples was then adjusted to $60 \%$ of the field holding capacity $(w / w)$ by adding deionized water. The soils were incubated at $25 \pm 1{ }^{\circ} \mathrm{C}$ in the dark. In the water anaerobic condition, added $2 \mathrm{~cm}$ of water on soils.

Fifty grams of sediment (dry weight) and water were added to each test chamber, the resulting water/sediment volume ratio was $4: 1$, and the sediment layer height was $2.5 \pm 0.5 \mathrm{~cm}$. The aerobic and anaerobic sediment/water

Table 2 Characteristics of soil and water-sediment

\begin{tabular}{|c|c|c|c|c|c|c|c|c|c|}
\hline Sample & Sample type & Site & $\mathrm{pH}$ & $\mathrm{OM}^{\mathrm{a}}(\%)$ & Clay (\%) & Sand (\%) & Silt (\%) & 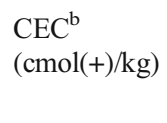 & $\begin{array}{l}\text { Physical clay } \\
\text { content } \\
(<0.01 \mathrm{~mm}) \mathrm{V} \%\end{array}$ \\
\hline \multirow[t]{4}{*}{ Soil } & Sierozem & Shanxi & 8.12 & 1.55 & 5.14 & 24.34 & 70.52 & 19.37 & - \\
\hline & Black soil & Ha'erbin & 7.43 & 5.14 & 7.22 & 21.87 & 70.91 & 22.47 & - \\
\hline & Krasnozem & Changsha & 5.44 & 1.33 & 3.21 & 27.43 & 69.36 & 11.21 & - \\
\hline & Fluvo-aquic soil & Langfang & 7.51 & 1.15 & 3.21 & 35.56 & 61.23 & 12.72 & - \\
\hline \multirow[t]{3}{*}{ Water/sediment } & Northeast Lake & Ha'erbin & 7.50 & 5.24 & - & - & - & 27.0 & 19.52 \\
\hline & Paddy field & Changsha & 5.42 & 1.33 & - & - & - & 14.2 & 29.28 \\
\hline & Reservoir & Beijing & 7.71 & 3.22 & - & - & - & 15.7 & 12.10 \\
\hline
\end{tabular}

${ }^{\text {a } O r g a n i c ~ m a t t e r ~}$

${ }^{\mathrm{b}}$ Cation-exchange capacity 
samples were acclimated for 14 days prior to the addition of the test substance, allowing the sediment conditions such as $\mathrm{pH}$ and redox potential to stabilize. Samples concentration in water phase was $10 \mathrm{mg} \mathrm{kg}^{-1}$ by adding $1000 \mathrm{mg} \mathrm{L}^{-1}$ cyflumetofen water stock solution with $0.5 \%$ acetone, and enough oxygen was input to the culture bottles. At the same time, an experimental system without tested pesticides was set up as control samples and used for microbial determination in the sediment and organic carbon in the water after the test. In order to avoid an adverse effect for the latent solvent on microbial activity in the test system, two acetone solvent systems were set up as control samples. Aqueous solution as the control group, other experimental conditions are consistent, used to measure of the proportions of hydrolysis and biodegradation in total degradation of water sediment systems. The incubation chambers were placed in an incubator at $25 \pm 1{ }^{\circ} \mathrm{C}$ and $60 \%$ humidity. In the anaerobic microcosms, the bottles were flushed with nitrogen gas to maintain anaerobic conditions. All tests were conducted in triplicate.

\section{Sampling, extraction, and chromatographic analysis}

Soil and sediment samples were sampled at $2 \mathrm{~h}, 1,2,4,8,12$, 20, 30, 40, 50, and 100 days, extracted using a previously published method (Li et al. 2013) with minor changes. Briefly, $5.0 \mathrm{~g}$ of the sample was weighed in a $50-\mathrm{mL}$ polypropylene centrifuge tube with a screw cap. Then, the sample was vortexed for $30 \mathrm{~s}$ and allowed to stand for $2 \mathrm{~h}$ at room temperature to distribute the pesticide evenly. Afterwards, $5.0 \mathrm{~mL}$ of acetonitrile was added, soils were added $2 \mathrm{~mL}$ Milli-Q water at the same time. The tube was shaken vigorously in a mechanical shaker for $3 \mathrm{~min}$ at $1300 \mathrm{rpm}$ at room temperature ensuring that the solvent interacted well with the entire sample. Subsequently, $3.0 \mathrm{~g}$ of anhydrous MgSO4 and $1.0 \mathrm{~g}$ of $\mathrm{NaCl}$ were added. The tubes were capped and immediately shaken for $1 \mathrm{~min}$ and then centrifuged for $5 \mathrm{~min}$ at $2811 \times \mathrm{g}$ relative centrifugal force. Next, $1.5 \mathrm{~mL}$ of the upper layer (ACN) was transferred into a single-use centrifuge tube containing $10.0 \mathrm{mg}$ of MWCNTs $(<8 \mathrm{~nm})$ sorbent. The tube was vortexed for $1 \mathrm{~min}$ and centrifuged for $5 \mathrm{~min}$ at $2400 \times \mathrm{g}$ relative centrifugal force. The resulting supernatant was filtered through a $0.22-\mu \mathrm{m}$ nylon syringe filter into an autosampler vial for UPLC-MS/MS injection.

Chromatographic separation was performed with a Waters Acquity UPLC binary solvent manager equipped with a Waters Acquity UPLC BEH C18 column $(2.1 \times 50 \mathrm{~mm}$, 1.7- $\mu \mathrm{m}$ particle size) (Milford, MA, USA). The mobile phases were used a previously published method (Li et al. 2013). The total analysis time was $4.0 \mathrm{~min}$. The column oven temperature was maintained at $45 \pm 1{ }^{\circ} \mathrm{C}$ to decrease viscosity, and the temperature of the sample vial holder was set at $5^{\circ} \mathrm{C}$. A triple quadrupole (TQD) mass spectrometer (Waters Corp., Milford, MA, USA) equipped with an ESI source was used to quantify these compounds. The nebulizer gas was $99.95 \%$ nitrogen, and the collision gas was $99.99 \%$ argon with a pressure of $3.2 \times 10^{-3}$ mbar in the T-Wave cell. All MS parameters were reported in our previous study (Li et al. 2013). MassLynx NT V.4.1 (Waters, USA) software was used for instrument control and data acquisition.

\section{Quality control and method validation}

In order to keep the quality and representativeness of the samples, several measures were used in the current study. In normal operation, soils and water/sediments were collected in different distinct sites. At least three replicate measurements were carried out in each sample analysis. Blank samples were systematically run before each sample analysis. Different quality parameters (linearity, recoveries, reproducibility, and sensitivity) were examined to evaluate the developed method. The linearity of the method was determined by analyzing the standard solutions and the different matrix-matched standard solutions in triplicate at seven concentrations, ranging from 0.005 to $2.0 \mathrm{mg} \mathrm{kg}^{-1}$, the correlation coefficients $\left(R^{2}\right)$ were greater than 0.9970 . For recovery assays, appropriate volumes of working standard solutions were added to blank samples. The spiked levels were $0.05,0.5$, and $2 \mathrm{mg} \mathrm{kg}^{-1}$. In the water, the average recoveries of cyflumetofen, B-1 and B-3 were 86.5-104.6, 81.8-99.5, and 90.5-104.7\%, the limits of detection (LODs) were $0.2,1.0$, and $0.3 \mu \mathrm{g} \mathrm{kg}^{-1}$, the limits of quantification (LOQs) were $0.6,3.4$, and $1.0 \mu \mathrm{g} \mathrm{kg}^{-1}$, respectively. In the soil, the average recoveries of cyflumetofen, B-1, and B-3 were 88.4-101.3, 89.3-106.3, and 90.1-101.1\%, the limits of detection (LODs) were $0.2,3.0$, and $0.5 \mu \mathrm{g} \mathrm{kg}^{-1}$, the limits of quantification (LOQs) were $0.7,9.8$, and $1.6 \mu \mathrm{g} \mathrm{kg}^{-1}$, respectively.

\section{Degradation kinetics and correlations}

The dissipation of cyflumetofen was fitted to simple firstorder kinetics (Beulke et al. 2000, Xuan-xiang et al. 2014). The corresponding rate constants, $\mathrm{k}$, and half-lives $\mathrm{t}_{1 / 2}$ (days) were calculated according the following equations:

$C_{t}=C_{0} e^{-k t}$
$t_{1 / 2}=\ln 2 / k$

In these equations, $t$ is the time elapsed since the pesticide was applied, $C_{t}$ is the pesticide concentration at time $t, C_{0}$ is the initial pesticide concentration immediately after the 
pesticide was applied (i.e., at $t=0$ ), $k$ is the dissipation coefficient, and $t_{1 / 2}$ is the time required for the pesticide concentration to reach half of the initial concentration (He et al. 2015, Koch et al. 2005).

Correlation analysis between soil properties and degradation half-lives of cyflumetofen were analyzed using the SAS software package (ver. 9.2, SAS Institute Inc., Cary, NC). Significant difference at $p<0.05$ between treatments was tested using a one-way analysis of variance (ANOVA) or $t$ test.

\section{Results}

\section{Degradation of Cyflumetofen in soils}

In aerobic environments, the half-lives of cyflumetofen in black soil, sierozem, krasnozem, and fluvo-aquic soil were $11.2,10.3,12.4$, and 11.4 days, respectively. According to the means, the degradation of cyflumetofen in these four soils was sierozem $>$ black soil $>$ fluvoaquic soil $>$ krasnozem. In water anaerobic conditions, the half-lives of cyflumetofen in black soil, sierozem, krasnozem, and fluvo-aquic soil were 13.1, 10.8, 13.9, and 12.8 days, respectively. The result of the degradation order was consistent with the aerobic environment, but in aerobic conditions the degradation rate was faster. The effects of different conditions and soil types on the half-lives of cyflumetofen were studied using a oneway ANOVA test with post hoc comparison (Tukey's test). It was shown that the differences in black soil, krasnozem, and fluvo-aquic soil were extremely significant difference $(p<0.05)$ under aerobic and water anaerobic conditions, the differences in sierozem were no significant difference $(p>0.05)$. And under the same conditions, the half-lives of cyflumetofen in the four soils were no significant difference $(p>0.05)$. The degradation dynamics of cyflumetofen were shown in Table 3, and the dissipation and analysis of cyflumetofen from four types of soil were shown in Fig. 1. According to Gavrilescu, the persistence of pesticides could be classified in terms of half-life as non-persistent (half-life $<30$ days), moderately persistent (half-life between 30 and 100 days) and persistent (half-life $>100$ days) (Gavrilescu 2005). Therefore, the results indicate that cyflumetofen is a non-persistent pesticide according to the classification mentioned earlier.

The degradation of the cyflumetofen in soils was mainly generated two metabolites. For example, in black soil in aerobic conditions and anaerobic conditions, the first day B-1 was generated, while, only $<0.24 \%$ of B-3 generated at the same time. With the extension of time, B-1 and B-3 gradually increased, cyflumetofen reduced gradually, until the degradation of cyflumetofen was greater than $95 \%$ for 100 days, at this time $>2.4 \%$ of B-1 and B-3 in the soil. In the aerobic conditions, B-1 and B-3 were generated faster than in the anaerobic conditions (Fig. 2a, b).

\section{Degradation of Cyflumetofen in water/sediment systems}

In aerobic environment, the half-lives of cyflumetofen in Northeast Lake, Hunan paddy field, and Beijng Shangzhuang reservoir were $15.4,16.9$, and 15.1 days, respectively. In anaerobic conditions, they were 16.5, 17.3, and 16.1 days, respectively. According to the means, the degradation of cyflumetofen in these three water/sediment systems was Shangzhuang reservoir > Northeast Lake $>$ Hunan paddy field. By analyzing the differences of the half-lives under aerobic and anaerobic conditions, the difference only in Shangzhuang reservoir was extremely significant difference $(p<0.05)$. And under the same conditions, the half-lives of cyflumetofen in the three water/sediments were also no significant difference $(p>0.05)$. The experiment was conducted in dark conditions, photolysis can be ignored and, therefore, the degradation was mainly hydrolysis and biodegradation. The proportion of hydrolysis and biodegradation of the total degradation of water sediment systems was shown in Fig. 3. With the extension of incubation, the proportion of hydrolysis was rising gradually. In the Northeast Lake and Beijng Shangzhuang reservoir water/sediment systems, it reached the maximum by 12 days. In the Hunan paddy field, it reached the maximum by 30 days, before there was a decrease. However, biodegradation is the main source of pesticide degradation, the proportion of hydrolysis and biodegradation was evaluated by difference between the aqueous solution and water/ sediments systems, assuming that biodegradation is caused by sediments microbes. Under anaerobic conditions, the proportion of biodegradation compared to total degradation is smaller compared with aerobic conditions. The proportion of hydrolysis in three water/sediment had minimal difference.

Similar to soil degradation, in the water sediment systems, cyflumetofen was transformed to metabolites B-1 and B-3. For example, in the Northeast Lake of aerobic conditions and anaerobic conditions, from the first day about $0.24 \%$ of B-1 and B-3 were generated. With the increasing time, B-1 and B-3 gradually increased, in water mainly produced B-3, until to $100 \mathrm{~d}$ there were $2.4-3.1 \%$ of B-1 and B-3 existed. Under the aerobic conditions, B-1 and B-3 were generated faster than in the anaerobic conditions (Fig. 2c, d). 
Table 3 The degradation dynamics of cyflumetofen and analysis

\begin{tabular}{|c|c|c|c|c|c|c|}
\hline $\begin{array}{l}\text { Soil } \\
\text { Water-Sediment }\end{array}$ & $\begin{array}{l}\text { Regression } \\
\text { equation }\end{array}$ & $\mathrm{R}^{2}$ & $\begin{array}{l}\text { Half-life } \\
\text { (day) }\end{array}$ & $\begin{array}{l}\text { Condifence interval } \\
(95 \%)\end{array}$ & $P$ & $t$ grouping \\
\hline Black soil $^{\mathrm{b}}$ & $\mathrm{Ct}=3.2767 \mathrm{e}^{-0.053 \times}$ & 0.8321 & 13.1 & $12.1431-14.5235$ & 0.0280 & A \\
\hline Black soil $^{\mathrm{a}}$ & $\mathrm{Ct}=2.4103 \mathrm{e}^{-0.062 \times}$ & 0.8401 & 11.2 & $10.6381-12.4819$ & & $\mathrm{~B}$ \\
\hline Sierozem $^{\mathrm{b}}$ & $\mathrm{Ct}=2.1219 \mathrm{e}^{-0.064 \times}$ & 0.8811 & 10.8 & $10.5069-10.9333$ & 0.0519 & $\begin{array}{l}\mathrm{A} \\
\mathrm{A}\end{array}$ \\
\hline Sierozem $^{\mathrm{a}}$ & $\mathrm{Ct}=2.0062 \mathrm{e}^{-0.067 \times}$ & 0.8609 & 10.3 & $10.0697-10.4000$ & & A \\
\hline Krasnozem $^{\text {b }}$ & $\mathrm{Ct}=3.8000 \mathrm{e}^{-0.050 \times}$ & 0.8458 & 13.9 & $13.4856-14.8478$ & 0.0030 & A \\
\hline Krasnozem $^{\mathrm{a}}$ & $\mathrm{Ct}=4.1780 \mathrm{e}^{-0.053 \times}$ & 0.8331 & 12.4 & $11.9524-13.0076$ & & B \\
\hline Fluvo-aquic soil $^{\mathrm{b}}$ & $\mathrm{Ct}=3.3726 \mathrm{e}^{-0.054 \times}$ & 0.9052 & 12.8 & $12.4925-13.7742$ & 0.0028 & A \\
\hline Fluvo-aquic soil $^{\mathrm{a}}$ & $\mathrm{Ct}=1.8186 \mathrm{e}^{-0.061 \times}$ & 0.8365 & 11.4 & $11.0236-12.0164$ & & $\mathrm{~B}$ \\
\hline Northeast Lake $^{c}$ & $\mathrm{Ct}=3.7903 \mathrm{e}^{-0.042 \times}$ & 0.8639 & 16.5 & $15.0928-17.2405$ & 0.3467 & $\begin{array}{l}\mathrm{A} \\
\mathrm{A}\end{array}$ \\
\hline Northeast Lake ${ }^{\mathrm{a}}$ & $\mathrm{Ct}=3.1979 \mathrm{e}^{-0.045 \times}$ & 0.8092 & 15.4 & $14.7682-16.4318$ & & $\mathrm{~A}$ \\
\hline Paddy field ${ }^{c}$ & $\mathrm{Ct}=5.3292 \mathrm{e}^{-0.040 \times}$ & 0.8433 & 17.3 & $16.2962-17.9704$ & 0.6092 & $\begin{array}{l}\mathrm{A} \\
\mathrm{A}\end{array}$ \\
\hline Paddy field ${ }^{\mathrm{a}}$ & $\mathrm{Ct}=6.3211 \mathrm{e}^{-0.041 \times}$ & 0.8381 & 16.9 & $16.2516-17.5484$ & & A \\
\hline Reservoir $^{\mathrm{c}}$ & $\mathrm{Ct}=3.3158 \mathrm{e}^{-0.043 \times}$ & 0.8836 & 16.1 & $15.6495-16.9505$ & 0.0147 & A \\
\hline Reservoir $^{\mathrm{a}}$ & $\mathrm{Ct}=5.3127 \mathrm{e}^{-0.046 \times}$ & 0.8131 & 15.1 & $14.6562-15.6638$ & & B \\
\hline
\end{tabular}

${ }^{\mathrm{a}}$ Aerobic conditions

${ }^{\mathrm{b}}$ Water anaerobic conditions

${ }^{\mathrm{c}}$ Anaerobic bottle conditions
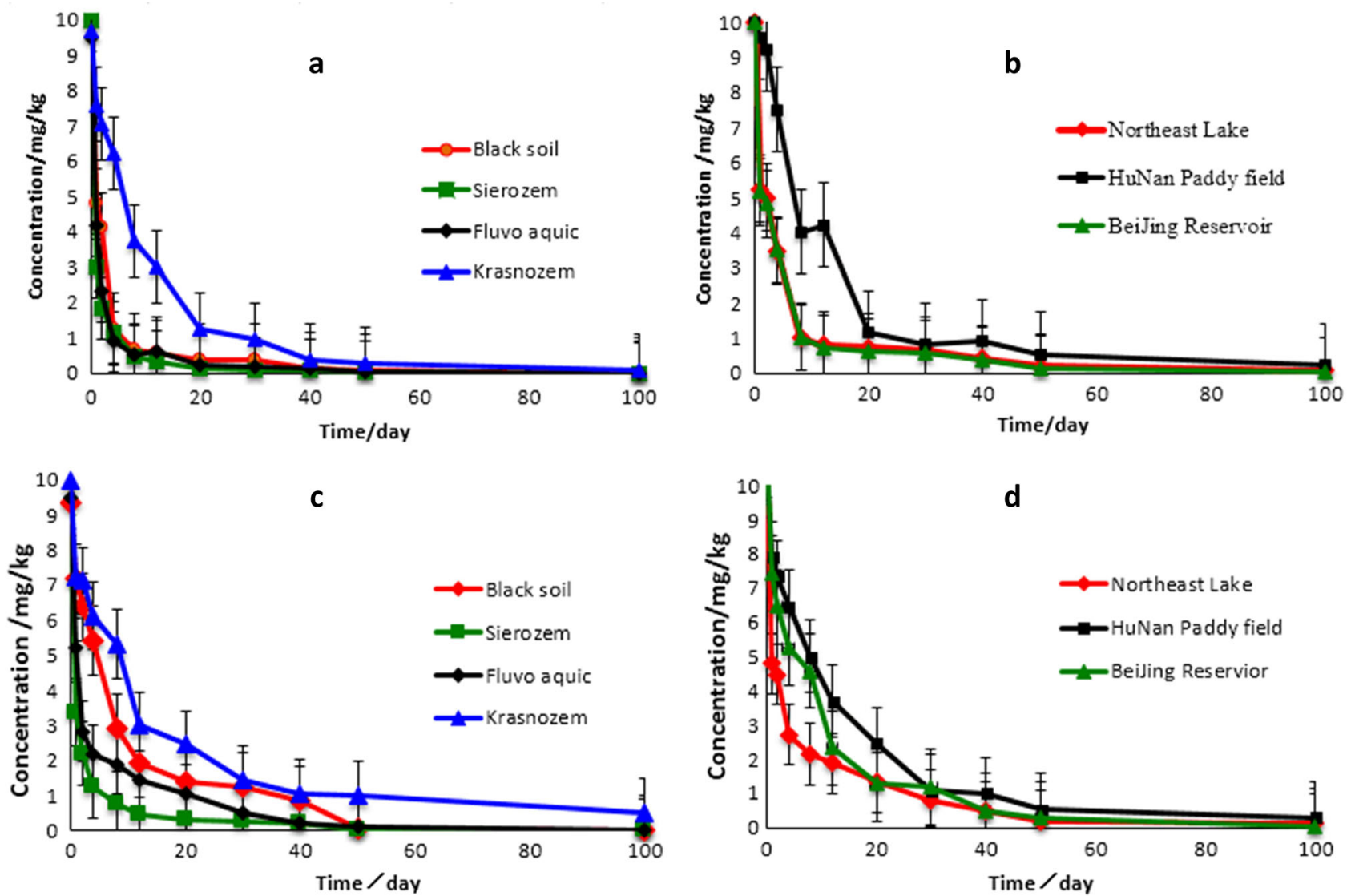

Fig. 1 The dissipation of cyflumetofen from four types of soil and three water/sediment systems (a and $\mathbf{b}$ were in the aerobic conditions, $\mathbf{c}$ and $\mathbf{d}$ were in the anaerobic conditions) 

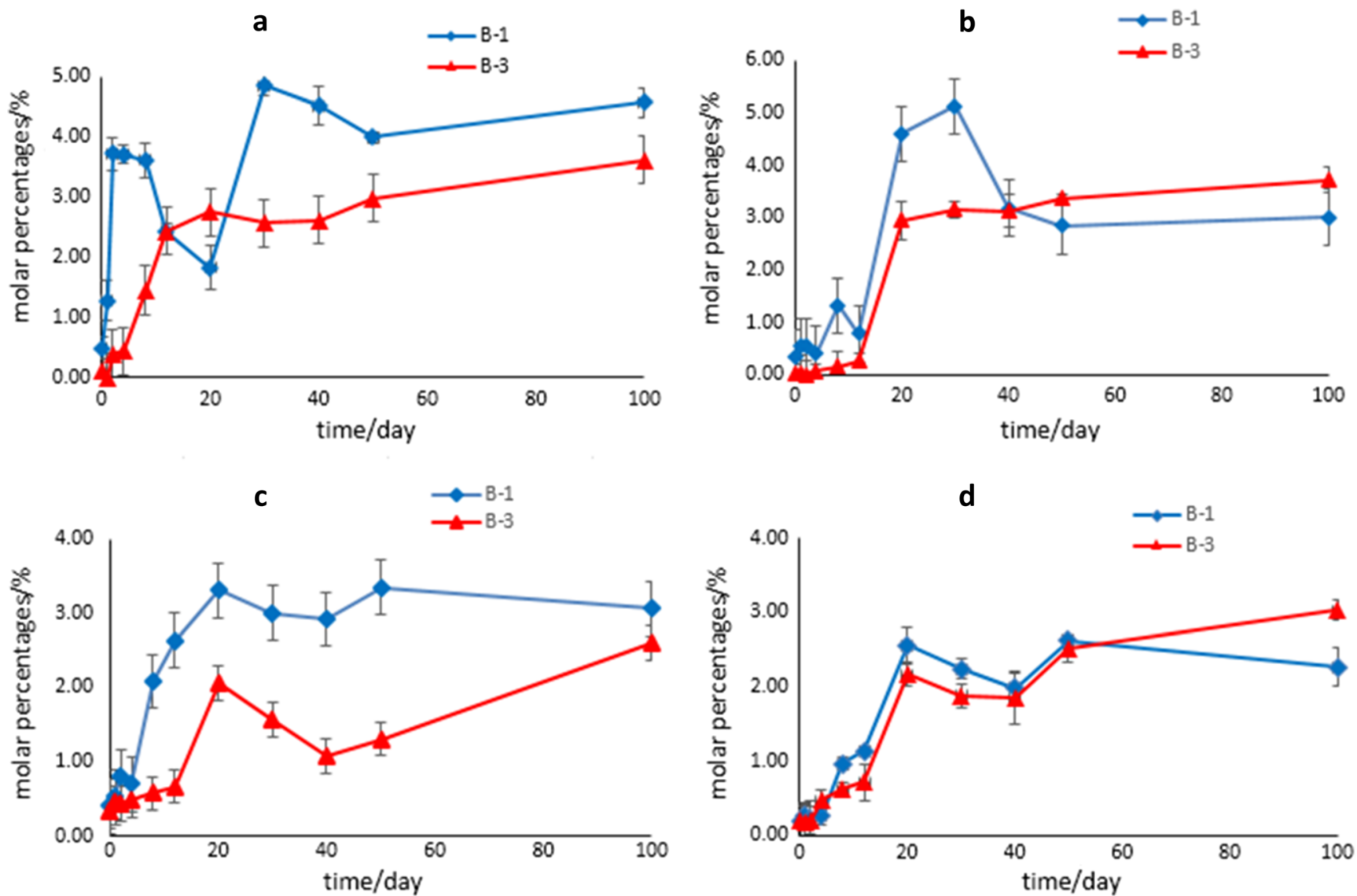

Fig. 2 The formation of main metabolites B-1 and B-3 (a and b were black soil in aerobic conditions and anaerobic conditions, respectively. $\mathbf{c}$ and $\mathbf{d}$ were Northeast Lake in aerobic conditions and anaerobic conditions, respectively)

In brief, B-1 and B-3 accumulate up to $3-5 \%$ of the parent initial concentration, this level allowing to consider them as relevant within any risk assessment, this accumulation resulted from processes of formation
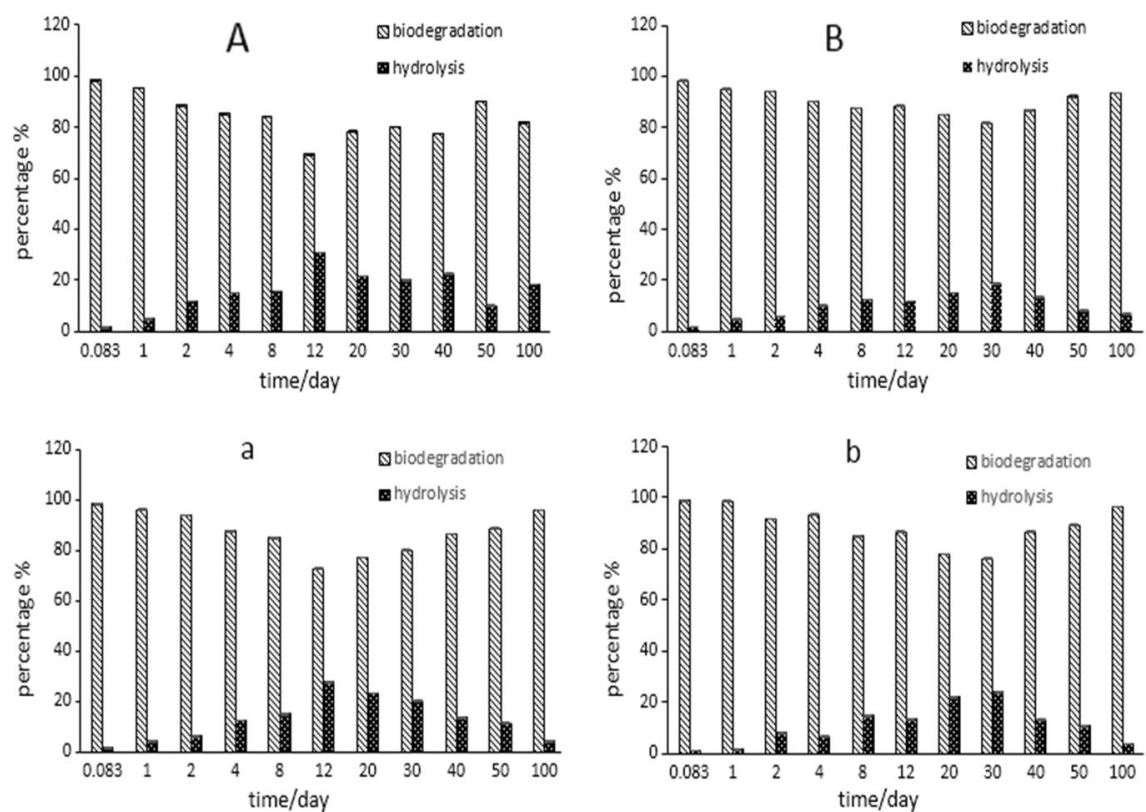

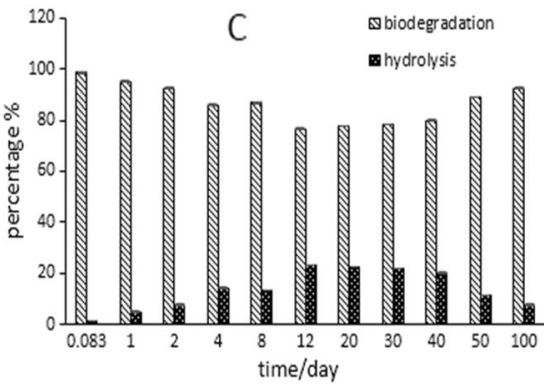

Fig. 3 The proportion of hydrolysis and biodegradation in total degradation of water sediment systems (A, B and C were in the aerobic conditions, a, b and $\mathrm{c}$ were in the anaerobic conditions, A and a were Northeast Lake, B and b were Hunan paddy field, C and c were Beijing Shangzhuang reservoir ) 
and degradation and, eventually, the metabolites degradation was likely to occur as PPDB database and EFSA indicated that those metabolites presented short halflives in soil (around 10 days).

\section{Discussion}

The behavior of a pesticide in the environment will be governed by complex physical, chemical, and biological processes, including sorption and desorption, volatilization, chemical and biological degradation, uptake by plants, surface runoff, and leaching (Wang et al. 2012). According to the PPDB, the half-life of cyflumetofen in the soil is 8 days and EU dossier Lab studies DT50 range 1.5-153.4 days, in our experiment, the results is reasonable. In the present study, cyflumetofen degraded faster in soil than in water/sediment $(p<0.05)$ and in aerobic conditions than in anaerobic, especially in black soil, krasnozem, fluvo-aquic and Shangzhuang reservoir $(p<0.05)$. Simple correlation between multiple variables analysis between half-life and selected soil properties indicated that cyflumetofen persistence in the four tested soils and three water/sediments was inversely connected to organic matter content, soil $\mathrm{pH}$ and cation-exchange capacity, positively correlated with sand. And there is a strong correlation between half-life and $\mathrm{pH}$, a weak relationship between halflife and organic matter content, cation-exchange capacity (Table 4). As we all know, ester hydrolyzes more thoroughly under the condition of alkaline, and under the condition of acid hydrolysis is a reversible process. Cyflumetofen as an ester, it is clearly that $\mathrm{pH}$ effect is significant, and it is also known in the PPDB database, $\mathrm{pH}$ can also effect soil properties such as electric charge and ionic strength (Clausen \&Fabricius 2001), thereby influencing the degradation rate of the pesticide (Arias-Estévez et al. 2008). It is generally accepted that soil-absorbed pesticides are thought to be unavailable for biodegradation (Ogram et al. 1985), as some studies suggested that compounds which were absorbed could

Table 4 Correlations study between half-lives and sample properties

\begin{tabular}{cccccc}
\hline Item & \multicolumn{2}{l}{ Soil } & & & \multicolumn{2}{l}{ Water/sediment } \\
\cline { 2 - 3 } \cline { 5 - 6 } & $\mathrm{r}$ & $\mathrm{p}$ & & $\mathrm{r}$ & $\mathrm{p}$ \\
\hline $\mathrm{pH}$ & -0.94333 & 0.0567 & & -0.99733 & 0.0466 \\
$\mathrm{OM}$ & -0.14521 & 0.8548 & & -0.76551 & 0.4450 \\
Clay & -0.50127 & 0.4987 & & - & - \\
Sand & 0.29036 & 0.7096 & & - & - \\
Silt & -0.16887 & 0.8311 & & - & - \\
CEC & -0.69726 & 0.3027 & & -0.45717 & 0.6977 \\
Physical clay & - & - & & 0.95854 & 0.1839 \\
\hline
\end{tabular}

$r$ correlation coefficients, $p$ probabilities be degraded by microorganisms (Park et al. 2001, 2002). Soil organic matter also adsorbs the molecule pesticides, organic matter content may also accelerate microbial activities (Getenga et al. 2004, Vidali 2001). But reported previously, microbial biomass was reduced under acidic $\mathrm{pH}$ (Houot et al. 2000). Therefore, these differences in degradation rate could be explained by the $\mathrm{pH}$ difference of the soils and water/ sediments as biotic and abiotic degradations are influenced by the soil properties, mainly $\mathrm{pH}$, temperature, clay, and organic matter contents (Salvia et al. 2014). However, no conclusion could be drawn and more experiments are required to understand this phenomenon.

\section{Conclusions}

The current work investigated, for the first time, the degradation of cyflumetofen in four soils and three water/sediment systems in China under aerobic and anaerobic conditions. The degradation dynamic of cyflumetofen followed firstorder kinetics. The half-lives of cyflumetofen were $<30$ days in the four soils and three water/sediments under aerobic and anaerobic conditions. And under the different conditions for the same samples, in the black soil, krasnozem, fluvo-aquic soil, and Beijng Shangzhuang reservoir, there were significant differences $(p<0.05)$. For the metabolites, in soils, cyflumetofen degraded B-1 and B-3, from the first day $0.24 \%$ B-1 was generated, while, only very low levels of B-3 generated at the same time. As time increased, B-3 gradually increased, cyflumetofen reduced gradually. Until 100 days, there were about 3.5\% B-1 and B-3 in the soils. In the water/sediment systems, from the first day, it degraded into B-1 in the sediment, and in the water mainly degraded into B-3. In the soil the B-1 and B-3 were generated faster than in water/sediment. Comprehensive soil and water sediment degradation behavior research has practical significance when evaluating its security according to the amount of cyflumetofen and its metabolites, B-1 and B-3. This research did not do studies of its metabolites about degradation, and further research is needed to clear the metabolites metabolic mechanism in the environmental. To provide the reference for related research.

Acknowledgments This work was supported by National Natural Science Foundation of China (31371970 and 31272070).

\section{References}

Arias-Estévez M, López-Periago E, Martínez-Carballo E, Simal-Gándara J, Mejuto J-C, García-Río L (2008) The mobility and degradation of pesticides in soils and the pollution of groundwater resources. Agric Ecosyst Environ 123:247-260 
Authority EFS (2012) Conclusion on the peer review of the pesticide risk assessment of the active substance cyflumetofen EFSA Journal (1), 77

Babić S, Horvat AJ, Pavlović DM, Kaštelan-Macan M (2007) Determination of $\mathrm{pK}$ a values of active pharmaceutical ingredients. TrAC Trends Anal Chem 26:1043-1061

Bermúdez-Couso A, Fernández-Calviño D, Pateiro-Moure M, NóvoaMuñoz JC, Simal-Gándara J, Arias-Estévez M (2011) Adsorption and desorption kinetics of carbofuran in acid soils. J Hazard Mater 190:159-167

Beulke S, Dubus IG, Brown CD, Gottesbüren B (2000) Simulation of pesticide persistence in the field on the basis of laboratory data - a review. J Environ Qual 29:1371-1379

Clausen L, Fabricius I (2001) Atrazine, isoproturon, mecoprop, 2, 4-D, and bentazone adsorption onto iron oxides. J Environ Qual 30:858869

Dalvie MA, Africa A, London L (2009) Change in the quantity and acute toxicity of pesticides sold in south African crop sectors, 1994-1999. Environ Int 35:683-687

Everett CJ, Matheson EM (2010) Biomarkers of pesticide exposure and diabetes in the 1999-2004 National Health and nutrition examination survey. Environ Int 36:398-401

Fenner K, Canonica S, Wackett LP, Elsner M (2013) Evaluating pesticide degradation in the environment: blind spots and emerging opportunities. Science 341:752-758

Gavrilescu M (2005) Fate of pesticides in the environment and its bioremediation. Engineering in Life Sciences 5:497-526

Getenga Z, Madadi V, Wandiga S (2004) Studies on biodegradation of 2, 4-D and metribuzin in soil under controlled conditions. Bull Environ Contam Toxicol 72:504-513

Hayashi N, Sasama Y, Takahashi N, Ikemi N (2013) Cyflumetofen, a novel acaricide - its mode of action and selectivity. Pest Manag Sci 69:1080-1084

He M, Jia C, Zhao E, Chen L, Yu P, Jing J, Zheng Y (2015): Concentrations and dissipation of difenoconazole and fluxapyroxad residues in apples and soil, determined by ultrahigh-performance liquid chromatography electrospray ionization tandem mass spectrometry. Environmental Science and Pollution Research, 1-9

Houot S, Topp E, Yassir A, Soulas G (2000) Dependence of accelerated degradation of atrazine on soil $\mathrm{pH}$ in French and Canadian soils. Soil Biol Biochem 32:615-625

Koch R, Burkness E, Hutchison W, Rabaey T (2005) Efficacy of systemic insecticide seed treatments for protection of early-growth-stage snap beans from bean leaf beetle (Coleoptera: Chrysomelidae) foliar feeding. Crop Prot 24:734-742

Li M, Liu X, Dong F, Xu J, Qin D, Zheng Y (2012) Determination of cyflumetofen residue in water, soil, and fruits by modified quick, easy, cheap, effective, rugged, and safe method coupled to gas chromatography/tandem mass spectrometry. J Sep Sci 35:27432749

Li M, Liu X, Dong F, Xu J, Kong Z, Li Y, Zheng Y (2013) Simultaneous determination of cyflumetofen and its main metabolite residues in samples of plant and animal origin using multi-walled carbon nanotubes in dispersive solid-phase extraction and ultrahigh performance liquid chromatography-tandem mass spectrometry. J Chromatogr A 1300:95-103

Löffler D, Römbke J, Meller M, Ternes TA (2005) Environmental fate of pharmaceuticals in water/sediment systems. Environmental Science \& Technology 39:5209-5218

Marcic D (2012) Acaricides in modern management of plant-feeding mites. J Pest Sci 85:395-408

McKinlay R, Plant J, Bell J, Voulvoulis N (2008) Endocrine disrupting pesticides: implications for risk assessment. Environ Int 34:168-183

MS Díaz-C, MJL d A, Barcelo D (2003) Environmental behavior and analysis of veterinary and human drugs in soils, sediments and sludge. TrAC Trends Anal Chem 22:340-351

OECD. Test 308: Aerobic and anaerobic transformation in aquatic sediment systems. In OECD Guidelines for the Testing of Chemicals; Organisation for Economic Cooperation and Development: Paris, 2002

Ogram A, Jessup R, Ou LT, Rao P (1985) Effects of sorption on biological degradation rates of (2, 4-dichlorophenoxy) acetic acid in soils. Appl Environ Microbiol 49:582-587

Park J-H, Zhao X, Voice TC (2001) Biodegradation of non-desorbable naphthalene in soils. Environmental science \& technology 35:2734 2740

Park J-H, Zhao X, Voice TC (2002) Development of a kinetic basis for bioavailability of sorbed naphthalene in soil slurries. Water Res 36: $1620-1628$

Pimentel D (1995) Amounts of pesticides reaching target pests: environmental impacts and ethics. J Agric Environ Ethics 8:17-29

Pimentel D, Levitan L (1986) Pesticides: amounts applied and amounts reaching pests. Bioscience 36:86-91

Salvia M-V, Experton J, Geandel C, Cren-Olivé C, Vulliet E (2014) Fate of pharmaceutical compounds and steroid hormones in soil: study of transfer and degradation in soil columns. Environ Sci Pollut Res 21: $10525-10535$

Seiber JN, Kleinschmidt LA (2011) Contributions of pesticide residue chemistry to improving food and environmental safety: past and present accomplishments and future challenges. J Agric Food Chem 59:7536-7543

Takahashi N, Nakagawa H, Sasama Y, Ikemi N (2012) Development of a new acaricide, cyflumetofen. J Pestic Sci 37:263-264

Vidali M (2001) Bioremediation. An overview. Pure Appl Chem 73: $1163-1172$

Wang L, Zhao P, Zhang F, Li Y, Du F, Pan C (2012) Dissipation and residue behavior of emamectin benzoate on apple and cabbage field application. Ecotoxicol Environ Saf 78:260-264

Xuan-xiang H, Mei-hong S, Wen-ying H, Yan-jun W, Han-yun K, Shuaifeng Z, Bureau JA, Protection HP (2014) Residue dynamics and safely applying technology of cyflumetofen in strawberry. Acta Agriculturae Zhejiangensis 6:029

Yoshida T, Ikemi N, Takeuchi Y, Ebino K, Kojima S, Chiba Y, Nakashima N, Kawakatsu H, Saka M, Harada T (2012) A repeated dose 90-day oral toxicity study of cyflumetofen, a novel acaricide, in rats. J Toxicol Sci 37:91-104 